pyogenes-aureus. Clinically they presented almost exactly the same appearances.

The types of $B$. diphtheriae found in cultures of some of these cases are shown in the photomicrograph. Granular forms were found in a number of cases; these organisms grew well on blood serum, and on this medium showed the typical morphology. They did not grow on ordinary lemco agar. In one or two instances, organisms which grew as granular types on blood serum appeared as solid forms on agar.

The wounds in which the infection was due to $B$. diphtheriae invariably began to clear up when antitoxin was administered, as was done in all cases. Such cases had shown no improvement whatever when an acid solution of hypochlorite (Lorain Smith), etc., was used alone. As soon as the body fluids became strongly antitoxic, improvement was noted. Experiments to determine the virulence of seven of the strains of $B$. diphtheriae causing these infections have been undertaken.

From the culture isolated in the case of P. E., a man suffering from an infected gunshot wound of the upper forearm, a toxin was produced, which in a dose of 0.5 c.c. killed a guinea-pig weighing $250 \mathrm{gm}$. in thirty-six hours. Another guinea-pig of the same weight was given a similar dose of this toxin, and in addition 1,000 units of antitoxin. The second guinea-pig was kept under observation for two weeks; it gained in weight and showed no paralyses. Several other strains tested showed somewhat less virulence.

The length of time required to clear up these infections varied considerably. One patient gave two successive negative cultures one week and a half after his infection was first determined, and he was given appropriate treatment. Twenty-three of the twenty-eight patients first observed had two successive negative cultures and were discharged from hospital in six

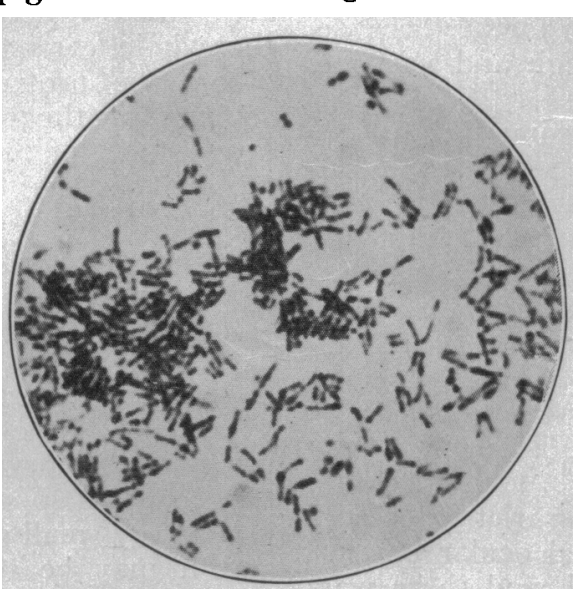

Fig. 3.-Culture of B. diphtheriae isolated il Patient P. E.: granular types especially numerous.

\section{CONICAL CORNEA, OR ANTERIOR MYOPIA *}

\section{EDWARD JACKSON, M.D. DENVER}

This subject, last brought before the section in the valuable symposium by Burnett, Sattler and Hermann Knapp, seventeen years ago, is of great scientific and practical interest, and our knowledge of it is still fragmentary. It has broad relations with other pathologic conditions of the eye and with more general problems of nutrition. The phrase "anterior myopia" is suggestive of relations to the larger class of posterior myopia and the process through which the great majority of cases of myopia develop. Both keratoconus and posterior or axial myopia arise from the pathologic distention of the sclerocorneal coat. In their production, states of general nutrition and local conditions both share, but to quite different extents. Axial myopia has been the subject of extended and thoughtful study, especially by our colleague, Dr. S. D. Risley. The succession of events, eye-strain, uveal congestion, scleral softening and distention, and passage of refraction from hyperopia to myopia have been often watched. Especially has astigma been noted, as an important factor in the production of eye-strain; the eyes passing, as Risley has phrased it, "from hyperopia to myopia through the turnstile of astigmatism."

The use of the eyes for near work is also recognized as a very general condition of the causation and progress of myopia. General diathetic diseases have also been considered to cause myopia. Batten $^{1}$ made a strong argument for the connection of myopia with general disease, and reported seventeen cases in support of this view, mostly of myopia arising in connection with cardiovascular disease. The share of general imand one half weeks or less. The average stay in isolation of these first twenty-three patients was thirty days. Five of the original twenty-eight patients still gave positive cultures more than six weeks after the nature of the infection was ascertained.

The treatment in all cases was practically the same. The men were isolated, were given diphtheria antitoxin, and their dressings were done with a rigid regard for asepsis. It has also been recommended that in future a routine bacteriologic examination of all suppurating wounds be made; also, that in no case should dressings of infected wounds be undertaken without rubber gloves being worn. It is regretted that because of the pressure of other work a Schick reaction could not have been done on all these cases.

Responsibility of Medical Staff.-The commanding officer of today, who is wise, instinctively acts according to the sentiment of Gen. Winfield Scott: "I am in the habit, myself, when on duty with troops, of paying great deference, and even of yielding my opinion, on matters deeply affecting health and life, to the advice of my medical staff."-Lieut.-Col. C. C. McCulloch, Jr., U. S. Army: The Scientific and Administrative Achievement of the Medical Corps of the United States Army, The Scientific Monthly. paired nutrition in causing myopia has not been so well demonstrated as the share of near eye work and strain from astigmia, but it seems necessary to assume such a factor to account for the fact that in many patients astigmia, eye-strain and retinochoroidal hyperemia leave undiminished hyperopia, while others become less hyperopic or myopic.

In the production of keratoconus the same factors may be concerned, but with far different relative importance. In anterior myopia, conditions of lowered general nutrition seem to be of first significance; anemia, acute general disease, chronic diathetic diseases, and impaired nutrition from many causes seem clearly associated with the beginnings of conical cornea. These may from the first cooperate with eye-strain, and later certain mechanical factors perpetuate and increase the trouble; but conical cornea rarely, if ever, begins when the patient is in robust general health. It is easy to see why the nonvascular cornea should especially suffer from lowered nutrition; while the most vascular portion of the sclera at the posterior

* Read before the Section on Ophthalmology at the Sixty-Eighth Annual Session of the American Medical Association, New York, June, 1917 .

1. Batten: Ophth. Rev., 1892, p. 1. 
pole of the eye should be most involved in a process, marked by active hyperemia. In axial myopia the brunt of the pathologic process falls near the posterior pole of the eye and near to the temporal side of the optic disk. The influences that produce keratoconus show little or no effect except toward the center of the cornea well removed from the vascular limbus. The beginnings of keratoconus are not closely associated with near work for the eyes, although after the defect has come into existence, and the eye is crippled for distant vision, the same vicious circle is established as in axial myopia; and the deterioration is

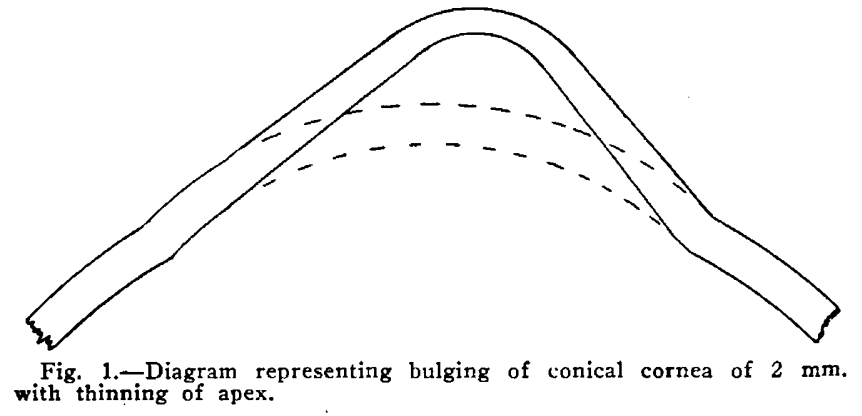

likely to be more rapid and to go much farther because of the presence in keratoconus of high and variable astigmia.

The conditions under which keratoconus begins are often difficult to determine. But in all cases that $\mathrm{J}$. have investigated carefully in this respect there has been a clear history of some antecedent severe impairment of nutrition.

One patient had suffered from extreme epistaxis; another from many attacks of epistaxis resulting in anemia; another had suffered from marked anemia from other causes, and three others first noticed defective vision while recovering from typhoid fever. One began after a severe attack of pleurisy. Others traced the beginning of the trouble clearly to scarlet fever and measles, although no history of special ocular inflammation at the time could be obtained, and there was no evidence of keratitis. A woman of 40 came out of two years of invalidism with astigmia of 5 and $6 \mathrm{D}$. incapable of perfect correction, although there was no evidence that her sight had been defective before that. A poorly nourished girl rapidly developed conical cornea at puberty.

This importance of generally depressed nutrition in causing keratoconus is also illustrated in the fact that most cases are bilateral, thirty-one out of forty-eight. Of the remaining seventeen, one patient had lost one eye probably from conical cornea, and the operations done for it; and in six others there was evidence that the eye, which was not counted as having conical cornea, had at one time been disturbed by the same process, but had soon regained a nutritive equilibrium retaining good vision with moderate astigmia. That is, of forty-eight cases, thirty-eight were really in the beginning instances of binocular disturbance. The importance of impairment of general nutrition in causing keratoconus seems certain.

\section{THE MECHANISM OF KERATOCONUS}

Plaut $^{2}$ reported a case of conical cornea in which the affected part of the cornea was greatly thickened, and experimentally produced in rabbits a condition resembling conical cornea in which the membrane was

2. Plaut: Klin. Monatsbl. f. Augenh., February, 1900, p. 65. greatly thickened. But the great mass of evidence indicates that thinning as well as softening of the tissue occurs so that it yields before normal or even diminished intra-ocular pressure.

Salzmann ${ }^{3}$ reports examination of a keratoconic eye in which the thinnest part of the cornea, the apex of the cone, was $0.17 \mathrm{~mm}$. in thickness, one-fourth or one-fifth the normal. He cites seven other reports of similar observations in which the apex of the cornea was found thinned to one-half or one-third the normal thickness. The ease with which the highly conical cornea can be indented by the lid margin, or altered in shape by lid pressure, makes it certain that the essential change in keratoconus includes thinning of the cornea with distention.

The actual protrusion in a case of conical cornea is generally much less than commonly occurs in posterior myopia. A protrusion of $1 \mathrm{~mm}$. makes a very marked conicity. My notes show no case of protrusion of the cornea of over $2 \mathrm{~mm}$. Yet that lengthening of the anteroposterior axis of the eyeball would cause only $6 \mathrm{D}$. of myopia. Anterior myopia is to no important extent axial. It is preeminently myopia of curvature (Fig. 1). The protrusion rarely involves the whole cornea. It often affects little more than one-half the area. In a few chronic cases of gradual development the whole cornea is altered in shape, and in some others the protrusion blends so gradually into the conicity of the cornea that the appearance suggests involvement of the whole cornea in the pathologic distention. Often the pathologic bulging is distinctly marked off from the normal corneal curve. Generally the apex of the cone is situated below and to the nasal side of the center of the cornea, often as much as 2 or $3 \mathrm{~mm}$. from the center. I have never noted it exactly at the center. This eccentric bulging gives the high astigmia that marks all cases of keratoconus. The center of the protrusion may present a fairly regular spherical curve, although usually it does not. But this center is so small that the light admitted through it is a very small proportion of that entering the pupil; and it may be so enormously myopic as to be far beyond the limits of correcting glasses or any possible usefulness in vision.

\section{ASTIGMIA}

Generally $\mathrm{th}$ e apex of the cone does not come in front of the contracted pupil. The patient is co mpelled to see through one side of the cone and so has to contend with

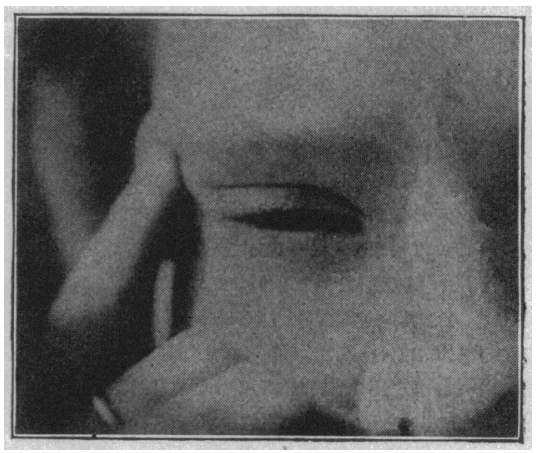

Fig. 2.-Corneal curvature modified by lid pressure produced by traction with tip of finger near the outer canthus.

high astigmia. In the direction of a line radiating from the apex of the cone, the cornea is relatively or actually flattened. In a direction at right angles to this, the tangent of a circle having the apex as a center, the curvature is progressively increased as the apex is approached. Thus. arises the high regular astigmia which with irregular astigmia characterizes the refraction of conical cornea. Of forty-five cases of keratoconus given optical correction, all had marked astigmia. The amounts of

3. Salzmann: Arch. f. Ophth. (Graefe's), 67, 1. 
regular astigmia noted in these seventy-nine keratoconic eyes are given in the accompanying table.

Almost as characteristic as the high degree of regular astigmia is its variability from day to day, or test to test, under variations of light; and its tendency to change from year to year, in amount, and to a less degree in direction. The astigmia as indicated by

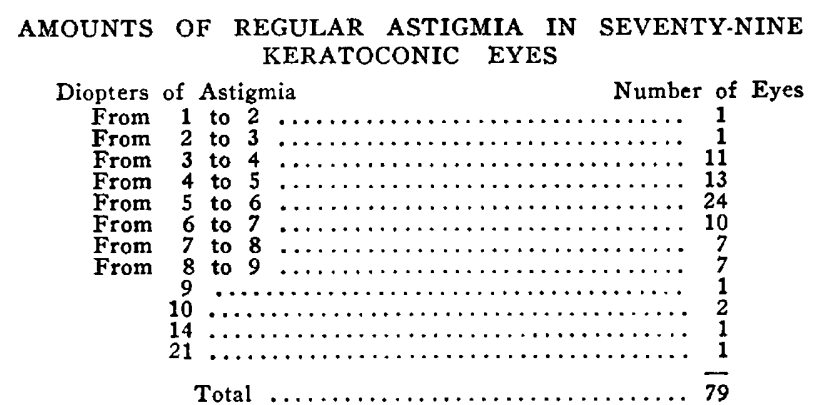

the glass preferred also varies greatly from the curvatures of the corneal meridians as shown by the ophthalmometer. Take the following case under observation thirteen years, while passing through college, medical school and hospital internship:

Case 1.-L. G. W., aged 19 when first seen

Nov. 6, 1903

Right, Ophthalmometer +4 . cy. axis $120^{\circ}$

Lens $-3.50 \mathrm{Sph} .=+2.50 \mathrm{cy}$. axis $153^{\circ}$

Left, Ophthalmometer $\mp 3.50 \mathrm{cy}$ axis $60^{\circ}$ Lens - 5. $=+3$. cy. axis $10^{\circ}$

Dec. 26, 1908:

Right, Ophthalmometer +4 cy. axis $140^{\circ}$ Lens $-3.25 \leftrightharpoons+0.75 \mathrm{cy}$. axis $160^{\circ}$

Left, Ophthalmometer +4 . cy. axis $65^{\circ}$ Lens $-4.50+2$. cy. axis $5^{\circ}$

Aug. 29, 1910:

Right, Ophthalmometer $-4.50 \mathrm{cy}$. axis $130^{\circ}$ Lens -3.75 $=+3$. cy, axis $170^{\circ}$

Left, Ophthalmometer $+4.45 \mathrm{cy}$. axis $60^{\circ}$ Lens $-4.50=+2$. cy axis $5^{\circ}$

Feb. 28, 1914: This patient's refraction changed with the use of pilocarpin

Right, without: -3.75 cy. axis $86^{\circ}=\% 1 \%$ partly

With pilocarpin : $-0.50=-3.50 \mathrm{cy}$. axis $110^{\circ}=\%$ mostly

Left without: $-5.50=\mp 35$ axis $5^{\circ}=5 \%$ partly

With pilocarpin: $-5.50=+3.75$ axis $5^{\circ}=\frac{6}{2}$ mostly

March 2, 1914: Without pilocarpin:

Right $-0.50=-3$. cy. axis $95^{\circ}=5 / 12$ partly

Left $-5.50=+3.50 \mathrm{cy}$. axis $5^{\circ}=5 \%$ mostly

March 6, 1914:

Right -2.75 cy. axis $95^{\circ}=5 \%$ mostly

Left $-5 .=+3.25$ cy. axis $180^{\circ}=5$ mostly.

July 3, 1916: The eyes now became less variable and still required:

Right -3 . cy. axis $87^{\circ}$

Left -5. cy. $=+3.25$ cy. axis $180^{\circ}$

For reasons given below, the close correction of such astigmia, at least to the extent of securing the best vision, and the keeping of the correcting glasses closely adapted to the eye, is of great practical importance.

EFFECTS OF LID PRESSURE

The effort to get the best vision is instinctive and persistent. It prompts the patient to try all sorts of experiments. The presbyope goes through the "trombone performance." The patient with uncorrected astigmia twists his head until he looks through his glasses obliquely in a way that makes them give the greatest assistance; the myope with too weak glasses looks through them obliquely to increase their strength, getting all the theoretical disadvantages of a full correction with some very important practical ones added. The myope without glasses partly closes his eyes, to lessen the circles of diffusion, thereby getting from the Greeks the name for his defect, which it retains today.

The patient with keratoconus, especially if it be largely myopic, is likely to resort to similar pressure to overcome, so far as possible, the disadvantageous curvature of the cornea. The long recognized "nipping" of the lids not only narrows circles of diffusion; it can also alter to a marked degree the corneal curvature, especially when the cornea is thinned and softened.

Case 2.-Miss G. E. F., aged 34, could change the curvature of her cornea equivalent to $1 \mathrm{D}$. change of refraction without closing the lids enough to prevent watching the ophthalmometric images. The effect was always to diminish the curvature in the horizontal meridian and increase it in the vertical meridian. The latter, however, was neutralized by the stenopaic effect of narrowing the palpebral fissure. Her cylinders, which were

$$
\begin{aligned}
& \text { Right }-6.50 \text { axis } 120^{\circ} \\
& \text { Left }-11 \text { axis } 120^{\circ}
\end{aligned}
$$

varied as much as $1 \mathrm{D}$. either way in frequently repeated tests, and as much as 15 degrees in the directions of the meridians chosen. The ophthalmometer reading indicated

$$
\begin{aligned}
& \text { Right }-5 . \quad \text { cy. axis } 80^{\circ} \\
& \text { Left }-5.50 \text { cy. axis } 155^{\circ}
\end{aligned}
$$

at repeated observations.

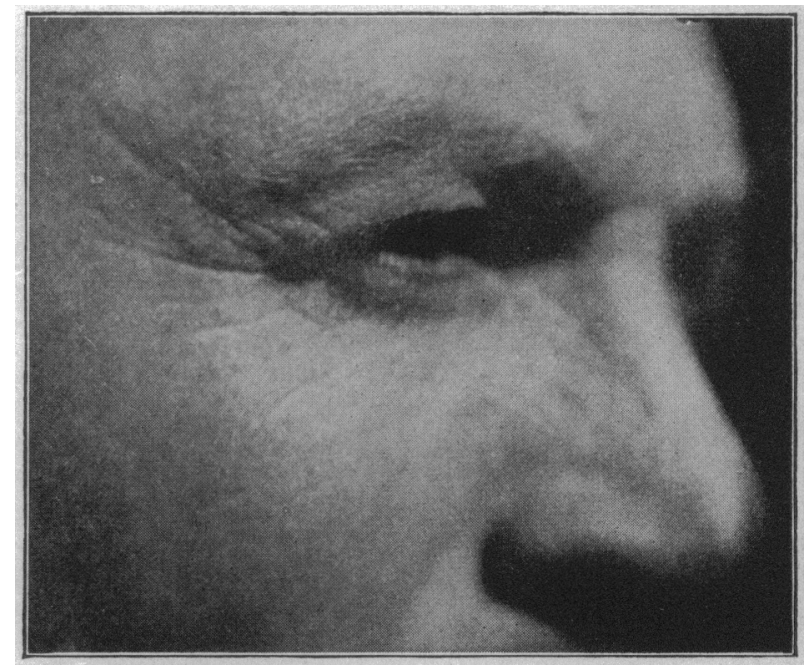

Fig. 3.-Face of patient at rest. Note wrinkles about the eye of a man of 27 .

Observations on other patients show that the usual effect of "nipping" the lids is that given above: to lessen the curvature in the direction of the palpebral fissure and increase it at right angles thereto, diminishing the myopia in the former and lessening the effect in the latter meridian. An unusual way of mak. ing such changes in the corneal curvatures is illustrated in the following case:

CASE 3.-A. M. H., a schoolgirl, aged 12, had the following vision and refraction:

Right $4 / 27$ partly -4 . sph. $=+1$. cy axis $95^{\circ}=4 / 4$

Left $4 / 100$ and with -9 . sph. $=4 / 20$

While not wearing glasses, she found she could bring up her vision so that she could see the blackboard by dragging on the outer canthus and upper lid of her right eye with the tip of a finger, as shown in Figure 2 . In this way she brought up vision to $4 / 7.5$ partly.

This patient was seen regularly at intervals of from twelve to thirty months, and by the time she was 19 her myopia had increased 2 diopters.

CASE 4.-K. F., man, aged 27 , in 1903, had scarlet fever when 14, and was sick for a year afterward, from which period he dated his poor sight, which had gradually increased. $\mathrm{He}$ 
had high conical cornea. His corneas were almost clear, showing a very slight general haze, and the ocular fundus was normal. The ophthalmometer showed:

$$
\text { Right }+7 \text {. cy. axis } 130^{\circ}
$$$$
\text { Left }+10 \text {. cy, axis } 80^{\circ}
$$

The following gave him the best vision:

$$
\begin{aligned}
& \text { Right }+5 \text {. sph. }=-8 \text {. cy. axis } 75^{\circ}=4 / 6 \\
& \text { Left }-6 \text {. cy. axis } 80^{\circ}=440
\end{aligned}
$$

He was not wearing glasses, having been unable to get any that helped him. Vision with either eye without lid tension was probably about $4 / 100$, but the instant his attention was directed to the letters, a curious distortion of the whole face occurred with lid tension that brought his vision up to $\$ / 20$. The change is shown in Figures 3 and 4.

The apex of the cone was below the edge of the $4 \mathrm{~mm}$. pupil in each eye, and the lower part of each pupil (half for the right and two-thirds for the left) was myopic. The upper part of each pupil was hyperopic. In his attempts to see (Fig. 4), the lower lid was pressed hard against the eyeball and stretched so that the edge of the lid went straight across the middle of the pupil. He was given correcting lenses, and warned of the importance of getting entirely out of the habit of compressing the eyeball with the lids.

At the end of eleven months he returned with corrected vision right $4 / 5$ and left $4 / 40$, and his corneas entirely clear. He had given up the habit of lid tension so completely that he could reproduce the effect very imperfectly and with special

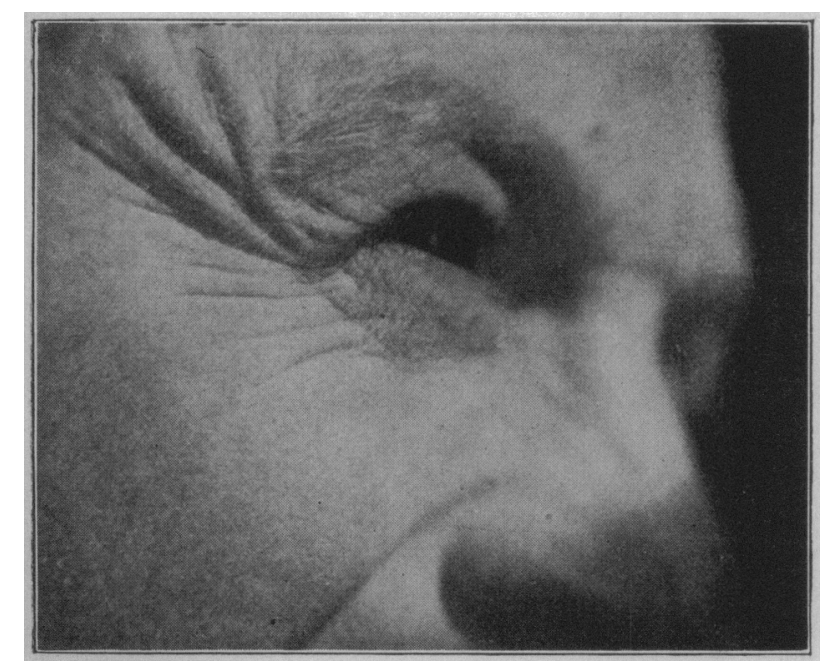

Fig. 4.-Face distorted by strong contraction of orbicularis to improve vision by lid pressure on the cornea.

effort. It was eight years before he could come again wearing the same glasses, which now required change, and the left eye had developed high myopia with choroidal absorption.

Right +4 . sph. $\asymp-8$. cy. axis $72^{\circ}=4 / 5$ partly

Left -4 . sph. $\asymp-8$. cy. axis $140^{\circ}=4 / 18$

Since than I have not seen him, but have heard from him that the sight of his left eye "is almost gone, and the right eye is in bad shape."

The following case shows how even the posterior distention of axial myopia may not save the cornea from protrusion, or how the malnutrition and softening of the coats may involve both the posterior and the anterior pole.

CASE 5.-B. G. M., girl, aged 15 , a bright student devoted to music, and strenuous in all she undertook, but anemic and "nervous," was brought to me for her eyes, Sept. 21, 1916. She had been wearing glasses since 8 years old, and now required them strengthened to:

Right $+0.75 \mathrm{sph} .-1.25 \mathrm{cy}$. axis $175^{\circ}=4 / 4$ plus

Left $+0.62 \mathrm{sph} .=-1.25 \mathrm{cy}$. axis $5^{\circ}=4 / 4$ plus

There was little choroidal disturbance, but the optic disks were red and slightly hazy. The ophthalmoscopic readings were:

Right $+1.50 \mathrm{cy}$, axis $90^{\circ}$ added to $43.5 \mathrm{D}$.

Left +2 cy. axis $90^{\circ}$ added to 44 . D.
There was some conjunctival hyperemia, burning and itching, in addition to the headache for which she came. She was under my care for four months and left with all symptoms relieved. June 19,1912, she returned, having been under good professional care in Chicago and elsewhere. The strenuous educational process had been supplemented by social engagements. The ophthalmometer now showed:

Right $+1.25 \mathrm{cy}$. axis $90^{\circ}$ added to 44 . D.

Left +10 . cy. axis $100^{\circ}$ added to 49 . $D$

The left eye was clearly suffering from conical cornea. A -10 . cy. axis $10^{\circ}$ gave it vision of $4 / 50$.

She next came, Sept. 14, 1912. The ophthalmometer showed : Left $+9.50 \mathrm{cy}$. added to 52 . D.

Lens, Left +3 . sph. $=-16$. cy. axis $145^{\circ}=4 / 15$ partly.

No marked change in the right eye, but

Right $+0.62 \mathrm{sph} .=-1.12 \mathrm{cy}$. axis $168^{\circ}=4 / 5$ partly.

Oct. 4, 1916, she caine once more broken down in health, highly anemic, listless, hopeless, and almost blind. She had been under treatment by various oculists in other cities. There was a history of operations, corneal ulcers, and finally enucleation of the left eye. Vision in the right was less than $1 / 100$; she was wearing a compress on it much of the time, and using eserin. It had suffered from an "ulcer" last winter. The ophthalmometer showed but little regular astigmia with a corneal curve of over $70 \mathrm{D}$., corneal radius about $5 \mathrm{~mm}$. $\mathrm{A}-30 \mathrm{D}$. sph. lens gave vision of $\% 100$. There was a nebula near the center of the cornea. The use of a bandage was almost stopped, being occasionally used for pain, the eserin was continued, and everything done to build up the general health of the patient.

Jan. 11, 1917, the eye felt better, and the iris was of good color and structure. The ophthalmometer showed: +6 to $+8 \mathrm{cy}$. axis $80^{\circ}$, added to 60 or $65 \mathrm{D}$.

Hot bathing of the eye has been substituted entirely for the bandage. The eye feels worst in the morning. The patient is to keep the head elevated.

CASEs 6 and 7.-F. B., aged 18, and his sister, aged 17, both came for conical cornea, with the history that every member of the family wore glasses. They were both students, the brother then in college. In all three eyes the area of protrusion was comparatively small and situated below the center. The brother's correction and vision was:

Right $+4.50 \mathrm{sph} . \subsetneq-7$. cy. axis $90^{\circ}=4 / 4$ partly

Left +4 . sph. $こ-7$. cy. axis $100^{\circ}=4 / 15$ partly

After eight years, the patient living on a ranch, these are: Right $+3.50 \mathrm{sph} .=-7.50 \mathrm{cy}$. axis $90^{\circ}=4 / 5$ Left -5 . sph. -7.50 cy. axis $85^{\circ}=4 / 12$

The sister had only the right eye affected. She had vision: Right $+1 .=-4$. cy. axis $38^{\circ}=4 / 5$

Left +1 . sph. $=0.25 \mathrm{cy}$, axis $90^{\circ}$

After four years she showed:

Right +2 . sph. $\cong-5$. cy. axis $55^{\circ}=4 / 4$ partly Left $+1.75 \mathrm{sph} . \Xi-0.37 \mathrm{cy}$. axis $65^{\circ}=4 / 4$ plus

These patients have taken proper care of their eyes, although living about 200 miles away so that they could be seen only at long intervals. The same is true of the following case:

CASE 8.-Jan. 29, 1910, S. J. G., aged 19, high school student, dated his poor sight to typhoid fever five years before: The eyes had not grown worse since. Both corneas were conical, the apex of each cone being fairly central, and the reflections of the rings of Placido's disk fairly circular. The centers of the pupils showed with the ophthalmoscope a myopia of 20 to $30 \mathrm{D}$., and the margins of the dilated pupils in the direction of the radii hyperopia of 15 to $20 \mathrm{D}$. The ocular fundus was normal in each eye, so far as could be determined. The patient was put on the regular use of pilocarpin. Lenses given: Right -15 . sph. $モ-8$. cy. axis $20^{\circ}=4 / 30$

$$
\text { Left }-10 \text {. sph. } こ-5 \text {. cy. axis } 20^{\circ}=4 / 30
$$

$\mathrm{He}$ was seen six months later with vision: Right $4 / 60$, left $4 / 12$.

Sept. 12, 1914 :

$$
\text { Right }+3 . \subsetneq-6 \text {. cy. axis } 25^{\circ}=5 / 15 \text { partly }
$$

Left $+6 . \sqsubseteq-1$. cy. axis $180^{\circ}=\% / 15$

Jan. 26, 1917: He comes several hundred miles to be seen once. 
Left shows keratitis, hazy with facet at apex of cornea $1 \mathrm{~mm}$. in diameter, no staining with fluorescein. He has completed school and college, and is now teaching school. He has used the pilocarpin continuously, but has not used his glasses sometimes during summer vacations.

In this paper no attempt has been made to give any complete account of conical cornea or complete histories of any of the forty-eight cases on which it is based. All of these cases have shown marked conicity of the cornea in some projection, asymmetrical except in Case 8 . None of them has shown any marked choroidal disease or atrophic crescent except Cases 1 and 2. In the series of cases from which the fortyeight were taken, there were thirty-nine cases of myopia with $3 \mathrm{D}$. or over of astigmia, and seventeen cases of hyperopia, with astigmia of $3 \mathrm{D}$. or over. None of the forty-eight patients, except Case 5, was subjected to operation, and all the worst cases have been included above.

The points to which attention is directed are:

1. Keratoconus arises from yielding of the cornea to intra-ocular pressure during a period of impaired nutrition, commonly due to general disease.

2. It gives rise to curvature ametropia, about which the ophthalmometer and the shadow test give little information of value for the selection of glasses.

3 . The subjective tests with lenses give widely variable results; and a decision as to the best lens is reached only after many trials, under varied conditions influencing the pupil.

4. It is extremely important that glasses should give the best vision under the conditions under which they will be used without lid pressure, which is to be carefully avoided.

5. The treatment should include:

Every effort to build up and sustain nutrition.

The continuous use of a miotic in the worst cases, usually pilocarpin.

The avoidance of softening the cornea by any form of bandages.

Under proper care operative treatment will rarely be needed.

\section{A NEW OPERATIVE METHOD FOR THE RELIEF OF ADVANCED CASES OF KERATOCONUS}

\author{
WITH REPORT OF TWO CASES * \\ MEYER WIENER, M.D. \\ sT. LOUIS
}

Conical cornea was observed by Demours as early as 1747 , and has been described by many authors since that time. There has never been any satisfactory explanation as to its production or cause. It is known that it is more frequent in females than in males, and is generally first noticed between the twelfth and fourteenth years of life. The probability is that some disturbance of internal secretion is the most potent causative factor.

Many operations have been devised for the relief of this condition, none of which has given entirely satisfactory results. The earlier surgical attempts to relieve conical cornea were bent toward changing the refraction of the eye behind the cornea rather than to alter the cornea itself. In 1817, Sir William Adams advised

* Read before the Section on Ophthalmology at the Sixty Eighth Anmual Session of the American Medical Association, New York, June, 1917. breaking up of the crystalline lens by needling. Tyrrell suggested a still different procedure, that of performing a peripheral iridectomy, on the theory of there being less deformity toward the margin of the cornea than in the center, and consequently a peripheral pupil would be of advantage. Crichett modified this a little later by permitting the iris to prolapse, and tying a ligature around it, thts distorting the pupil. Frequent puncturing of the periphery of the cornea has also been resorted to for many years. Von Graefe suggested the greatest advance for the relief of this condition by proposing that the epithelial coat of the apex be shaved off and then cauterized with silver nitrate; finally the floor of the resulting ulcer was punctured, the object being to try to bring about scar contraction with resulting flattening. Bowman had previously excised a disk of corneal tissue from the apex with a trephine, but without any great success. A number of cases in which he excised an elliptical piece were reported by Bader. To prevent prolapse of the iris he passed horse-hair sutures through the cornea, previous to making the excision, a fterward tying them over the edges of the wound. This operation has probably been the one most frequently advocated of those procedures which we might term purely surgical. The
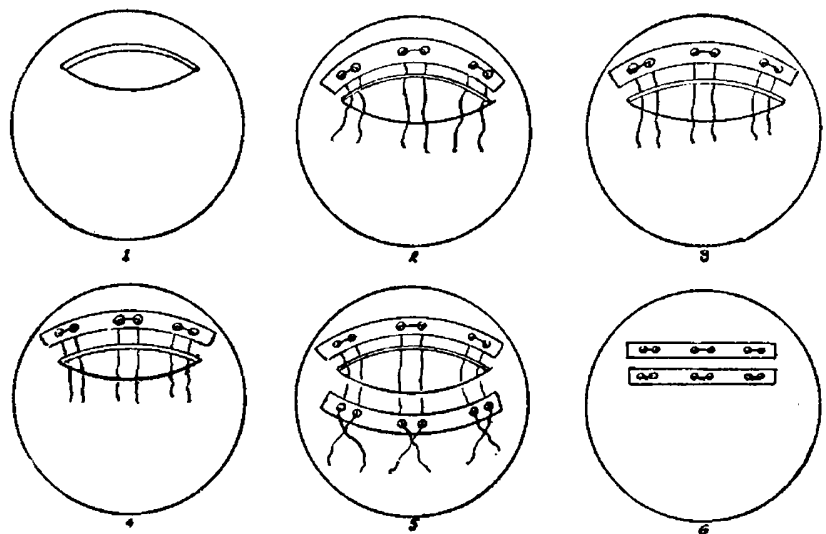

Details of operation: 1. Elliptic incision of cornea near margin. 2. Gold strip with double-armed sutures already in place ready to be passed through peripheral margin of cut. 3. Sutures passed through peripheral margin of wound. 4. Sutures passed through central margin
of wound. 5. Sutures passed through second gold strip. 6. Gold strips in place after completion of operation.

use of the electric cautery, or frequent punctures of the apex with a needle, being more simple and less dangerous, are the methods most often used today, when anything at all is advised. Recently a case of marked flattening has been reported from the use of the high frequency current applied to the apex of the cone.

The great objection to excision of the apex of the cone, or of a peripheral part of the cornea, has been the almost invariable prolapse of the iris into the wound with the sudden gush of aqueous, with its resulting infection, or irritation from an extensive leukoma adherens. The method which I am proposing is an excision of the corneal tissue in such a manner as to avoid iris prolapse. This I have succeeded in doing, and with fairly good visual results.

As the cornea near the apex of the cone is so thin as to preclude the probability of a successful dissection, I have elected to excise a segment near the periphery, as best suited to the method. The idea is to excise a segment of the cornea, including as nearly as possible its entire thickness, without puncturing Descemet's membrane, and then suture the wound. Necessarily there as an unusual tension on the stitches 